\title{
Trombocitopenia adquirida e cirurgia cardíaca: relato de caso
}

\author{
Hélcio GIFFHORN*, Amândio RAMPINELLI*, Lourival BONATELLI FILHO*, Jauro COLLAÇO*
}

RBCCV 44205-587

Giffhorn H, Rampinelli A, Bonatelli Filho L, Collaço J - Trombocitopenia adquirida e cirurgia cardíaca: relato de caso. Rev Bras Cir Cardiovasc 2002; 17(2): 58-62.

RESUMO: Trombocitopenia é um grande problema hemostático em pacientes submetidos à cirurgia cardíaca. Relataremos dois casos de pacientes com diminuição adquirida das plaquetas: um devido a uremia e outro por disfunção valvar (disfunção de prótese em posição mitral). Estes pacientes tiveram períodos de transoperatório e pós-operatório imediato sem intercorrência. $A$ perda sangüínea não foi maior do que a esperada. A transfusão de plaquetas realizada durante a operação pode prevenir futuras complicações na recuperação do paciente.

DESCRITORES: Trombocitopenia, cirurgia. Plaquetas, patologia. Coagulação sangüínea. Hemostasia.

\section{INTRODUÇÃO}

Alterações hemostáticas presentes no pré-operatório de operação cardíaca representam um grande risco cirúrgico porque elas podem aumentar após a operação com circulação extracorpórea. A disfunção plaquetária é o defeito hemostático mais comumente observado na fase de pós-operatório imediato. Atenção especial deve ser tomada se o paciente já apresenta trombocitopenia no pré-operatório(1).

\section{RELATO DOS CASOS}

\section{Caso 1}

Paciente Q.T.O., branco, do sexo masculino, 70 anos de idade, portador de bioprótese mitral e fibrilação atrial crônica, chegou ao hospital em insuficiência cardíaca (NYHA III). O exame físico revelava um sopro sistólico em focos mitral e tricúspide, estertores bolhosos de médias bolhas em campos médios pulmonares. As escleras estavam hipocoradas. Não havia outros achados ao exame físico. Exames laboratoriais: hemoglobina, 13,7g/dl; leucócitos, 9700/ $\mathrm{mm}^{3}$; contagem de plaquetas, $83000 / \mathrm{mm}^{3}$; parcial urina, normal; teste de Coombs, negativo; haptoglobinas, 82,6mg/dl; LDH, $896 \mathrm{U} / \mathrm{L}$; bilirrubina total, $1,44 \mathrm{mg} / \mathrm{dl}$; uréia, $72 \mathrm{mg} / \mathrm{dl}$ e creatinina, $0,96 \mathrm{mg} /$ dl.

O hematologista assistente ao caso concluiu que a origem da trombocitopenia era por destruição plaquetária pela prótese com disfunção. A contagem plaquetária imediatamente antes da operação era de $79000 / \mathrm{mm}^{3}$. O paciente foi submetido a operação para retroca da prótese valvar, sendo colocada uma nova prótese do tipo biológica porcina de número 31 . O tempo de circulação extracorpórea foi de 110 minutos e o tempo de oclusão aórtica de 71 minutos. A perda estimada de sangue no período transoperatório foi de $650 \mathrm{ml}$. Durante a operação foi usado ácido tranexâmico na dose de $2,0 \mathrm{~g}$. A drenagem no período pós-operatório imediato foi de $628 \mathrm{ml}$ e de $200 \mathrm{ml}$ no primeiro e segundos dias de UTI, respectivamente. $O$ paciente necessitou usar marcapasso temporário por 36 horas devido a ritmo juncional de resposta ventricular baixa. A contagem de plaquetas foi de $32000 / \mathrm{mm}^{3}$ imediatamente após a circulação extracorpórea e elevou-se a $87000 / \mathrm{mm}^{3}$ no primeiro dia pós-operatório. O paciente recebeu 10 unidades de concentrado de plaquetas após a circulação

Trabalho realizado no Instituto de Cardiologia do Estado de Santa Catarina. São José, SC, Brasil.

Recebido para publicação em junho de 2001.

*Do Instituto de Cardiologia do Estado de Santa Catarina.

Endereço para correspondência: Hélcio Giffhorn. Rua Gastão Câmara, 694 - apto. 1206 - Bigorrilho. Curitiba, Paraná, Brasil. CEP 80730-300. Tel: (41) 3221121 r.283. e.mail: cardiaca@ rla13.pucpr.br 
extracorpórea e na chegada à UTI. Ele recebeu alta hospitalar no 14으 dia de pós-operatório assintomático. A contagem plaquetária na alta hospitalar era de $127000 / \mathrm{mm}^{3}$.

\section{Caso 2}

Paciente Q.T.S., do sexo masculino, branco, apresentava diabete melito não insulino-dependente, gota, doença arterial coronariana e insuficiência renal crônica de origem vascular. A história clínica era angina de classe funcional II (CASS). O exame físico não apresentava particularidades. Não havia história clínica de hemorragias. Exames laboratoriais pré-operatórios: hemoglobina, 10,2g/dl; número de leucócitos, $4500 / \mathrm{mm}^{3}$; contagem de plaquetas, $99000 / \mathrm{mm}^{3}$; uréia, $102 \mathrm{mg} / \mathrm{dl}$; creatinina, $3,77 \mathrm{mg} / \mathrm{dl}$. O número de plaquetas imediatamente antes da operação era de $82000 / \mathrm{mm}^{3}$. O ecocardiograma com doppler demonstrou hipertrofia ventricular esquerda. O estudo cineangiocoronariográfico revelou lesões coronárias múltiplas (artéria coronária direita - lesões de 40 e $60 \%$; tronco de coronária esquerda - $50 \%$; artéria descendente anterior - $90 \%$ na origem e ocluída após o primeiro ramo diagonal; artéria circunflexa - com lesão de $40 \%$ na origem) associada à hipertrofia ventricular esquerda. A contractilidade miocárdica era normal e a manometria cardíaca apresentava hipertensão arterial sistêmica: aorta - 200/100/143mmHg e ventrículo esquerdo $-200 / 20 \mathrm{mmHg}$.

$\mathrm{O}$ número baixo de plaquetas era devido à insuficiência renal crônica. $O$ paciente foi submetido à operação de revascularização miocárdica (ponte de veia safena para o primeiro ramo diagonal e ponte de veia safena para o ramo circunflexo). O tempo de circulação extracorpórea foi de 41 minutos e 14 minutos de oclusão aórtica. Não foi empregado o ácido tranexâmico (reservado para os casos de reoperação em nosso Serviço) e foram transfundidas 10 unidades de plaquetas (aproximadamente $500 \mathrm{ml}$ ). A perda estimada de sangue no transoperatório foi de $1000 \mathrm{ml}$. A operação transcorreu sem problemas. A perda de sangue na primeira noite após a operação foi de $275 \mathrm{ml}$ e de $180 \mathrm{ml}$ no primeiro dia de pós-operatório. A contagem plaquetária foi de 69000 e $87000 / \mathrm{mm}^{3}$ nestes dias, respectivamente. O paciente não recebeu transfusão de sangue ou seus derivados. Ele permaneceu dois dias na UTI e recebeu alta hospitalar no 11ㅡ. dia pós-operatório assintomático. A contagem plaquetária na alta hospitalar era de $110000 / \mathrm{mm}^{3}$.

\section{COMENTÁRIOS}

A contagem normal de plaquetas varia de 150000 a $350000 / \mathrm{mm}^{3}$ e trombocitopenia ocorre quando o número de plaquetas está abaixo de $150000 / \mathrm{mm}^{3}$ (2). Os dois pacientes acima discutidos apresentavam anormalidades na contagem plaquetária de diferentes origens: lesão plaquetária por uma superfície vascular anormal (disfunção de prótese valvar) e uremia. No primeiro caso, a superfície irregular devido à disfunção da bioprótese em posição mitral foi a única causa identificável que poderia diminuir o número de plaquetas. A disfunção da bioprótese leva à ativação plaquetária com liberação dos corpos densos, numa seqüência similar a da ativação fisiológica( ${ }^{(3)}$. O segundo paciente apresentava insuficiência renal crônica e a uremia pode explicar a presença do menor número de plaquetas. O mecanismo exato desta alteração ainda não foi determinado ${ }^{(4)}$. Atualmente, a patogênese dos efeitos hemostáticos em pacientes urêmicos estaria relacionada a dois mecanismos: interação defeituosa da parede vascular com a plaqueta circulante e um fator de von Willebrand defeituoso (responsável pela aderência plaquetária).

A plaqueta torna-se ativada durante a circulação extracorpórea pelo contato com superfícies nãoendoteliais na máquina coração-pulmão e com produtos de granulócitos e células endoteliais ativadas durante a circulação extracorpórea. As plaquetas apresentam maior lesão quando usados oxigenadores de bolhas ${ }^{(1)}$. Outros fatores que também alteram o número de plaquetas após a operação cardíaca são o uso da hipotermia e do sulfato de protamina. Os dois pacientes estudados fizeram uso de oxigenadores do tipo membrana e "bio-pump" durante a circulação extracorpórea. Ocorre recuperação plaquetária normalmente somente após seis a oito horas da operação(1).

Alguns cuidados especiais devem ser tomados quando um paciente com plaquetopenia é encaminhado para operação cardíaca com circulação extracorpórea. O banco de sangue deve ser notificado para reservar concentrado de plaquetas para transfusão durante a operação e no pós-operatório imediato. O uso de "cell-saver" permite otimizar a recuperação do sangue (hemáceas lavadas) aspirado do campo operatório. O ácido tranexâmico é empregado em nosso Serviço somente em casos de reoperações, apesar do melhor efeito proporcionado pela aprotinina na proteção da função plaquetária contra o ataque da plasmina durante a circulação extracorpórea ${ }^{(5)}$.

Em cirurgia cardíaca, a causa mais comum de trombocitopenia pré-operatória é aquela relacionada a medicações usadas antes da operação. Diuréticos tiazídicos, quinidina, acetaminofen, acetazolamida, digitoxina, fenitoína, ácido etacrínico, alfa- metildopa, espironolactona, heparina, todos têm sido implicados em várias causas de trombocitopenia induzida por drogas, embora o mecanismo exato nem sempre te- 
nha sido bem definido.

A trombocitopenia induzida por heparina pode ser de dois tipos: I - causada pela interação direta entre heparina e as plaquetas ; II - causada por reação imune mediada por imunoglobulinas $\mathrm{G}^{(6-8)}$. Pacientes recebendo heparina apresentam estes efeitos numa incidência de 5 a 25\% dos $\operatorname{casos}^{(9)}$. O paciente do segundo caso clínico usava heparina por via subcutânea, mas ela não é considerada como uma causa de diminuição do número de plaquetas em pacientes ${ }^{(9)}$ (Quadro 1).
Nos pacientes a serem submetidos a circulação extracorpórea, podem ser realizadas avaliações imunológicas (teste de anticorpos contra heparina com o plasma do próprio paciente) ${ }^{(8,10)}$.

Para a anticoagulação alternativa a ser usada não há um consenso. À exceção do heparinóide danaparóide (ORG - 10172), as heparinas de baixo peso molecular podem apresentar reação cruzada em 80 a $90 \%$ dos $\operatorname{casos}^{(7,11-13)}$. Outras opções seriam o iloprost $^{(12,14,15)}$, o ancrod ${ }^{(16-18)}$, argatroban ${ }^{(19)}$, e a hirudina recombinante (20-23) - (Quadro 2).

\section{QUADRO 1}

TROMBOCITOPENIA INDUZIDA POR HEPARINA

\begin{tabular}{|ll|}
\hline DIAGNÓSTICO & \\
Trombocitopenia moderada & -20.000 a $100.000 / \mathrm{mcl}$ plaquetas I \\
Início após administração & - precoce: 1 a 3 dias \\
Avaliação de uso prévio heparina & - tardia: acima de 5 dias \\
\hline $\begin{array}{l}\text { TIPOS } \\
\text { I-contato direto heparina-plaquetas }\end{array}$ & INCIDÊNCIA \\
II-reação imune (IgG) & - mais freqüente \\
\hline $\begin{array}{l}\text { TESTES LABORATORIAIS PARA O DIAGNÓSTICO } \\
\text {-duas contagens plaquetárias abaixo de 150.000/mcl } \\
\text {-teste positivo para anticorpos IgG dependentes heparina }\end{array}$ & \\
\hline $\begin{array}{l}\text { MODO SENSIBILIZAÇÃO À HEPARINA } \\
\text {-endovenosa }\end{array}$ & \\
--subcutânea & \\
-uso cateter intra-arterial & - diálise \\
\hline
\end{tabular}

Referências Bibliográficas: 8,10,21,24

QUADRO 2

SUBSTITUTOS DA HEPARINA

\begin{tabular}{|c|c|c|c|c|c|c|c|c|}
\hline DROGA & $\begin{array}{l}\text { Tempo Ação } \\
\text { (Meia vida) }\end{array}$ & Neutralização & Complicações & Excreção & Controle Anticoagulação & Dose & Observações & Referências \\
\hline Iloprost & 15 minutos & Não & $\begin{array}{l}\text { Hipotensão } \\
\text { arterial }\end{array}$ & - & $\begin{array}{l}\text {-Tempo de sangramento; } \\
\text {-Fibrinopeptídio A } \\
\text {-Fator plaquetário } 4 \\
\text {-Agregação plaquetária ao ADP e heparina }\end{array}$ & $\begin{array}{c}10-30 \\
\mathrm{ng} / \mathrm{kg} / \mathrm{min}\end{array}$ & $\begin{array}{c}\text { Administração junto } \\
\text { à Heparina }\end{array}$ & $14,15,26$ \\
\hline Ancrod & $3-5$ horas & $\begin{array}{l}\text { Antiveneno } \\
\text { específico }\end{array}$ & Reação alérgica & Hepática & $\begin{array}{c}\text { Concentração de fibrinogênio - menor de } \\
0,7 \mathrm{~g} / \mathrm{l}\end{array}$ & $\begin{array}{c}1,65+-0,55 \\
U / g\end{array}$ & $\begin{array}{l}\text { - Agente } \\
\text { Fibrinogenolítico - } \\
\text { Iniciar antes CEC }\end{array}$ & $16,17,18$ \\
\hline Argatroban & 30 minutos & Não & $\begin{array}{l}\text { Cefaléia, dor, } \\
\text { tontura, astenia, } \\
\text { rash, náusea, } \\
\text { reação local infusão } \\
\end{array}$ & Hepática & $\begin{array}{l}\text { Controle: } \\
\text { - TCA } \\
\text { - KPTT }(1,5-3 x x / \text { controle) }\end{array}$ & $3 \mathrm{mcg} / \mathrm{kg} / \mathrm{m}$ & $\begin{array}{l}\text { Suspender } 30 \\
\text { minutos antes } \\
\text { operação }\end{array}$ & 19,25 \\
\hline $\begin{array}{c}\text { Hirudina } \\
\text { Recombinante }\end{array}$ & $\begin{array}{l}30-60 \\
\text { minutos }\end{array}$ & R-Meizotrumbina & $\begin{array}{c}\text { - Hemorragia } \\
\text { - Insuficiência renal } \\
\text { aguda }\end{array}$ & Renal & $\begin{array}{l}\text { Tempo coagulação ecarin (TCA II): } 180 \text { - } \\
200 \text { segundos }\end{array}$ & $\begin{array}{l}\text { Bolos: } \\
0,25 \mathrm{mg} / \mathrm{kg} \\
\text { Prime: } \\
0,20 \mathrm{mg} / \mathrm{kg}\end{array}$ & $\begin{array}{l}\text {-Inibição trombina } \\
\text {-Concentração } \\
\text { desejada } \\
3,5-4,0 / \mathrm{mg} / \mathrm{ml}\end{array}$ & $19,21,22,23$ \\
\hline $\begin{array}{l}\text { Heparinóides } \\
\text { (danapanóide) }\end{array}$ & 8 horas & Não & Hemorragia & Renal & nivel anti-fator $\mathrm{X}$ a & $1,5 \mathrm{UI} / \mathrm{ml}$ & $\begin{array}{l}\text { Heparina de baixo } \\
\text { peso molecular }\end{array}$ & $7,11,12$ \\
\hline
\end{tabular}


RBCCV 44205-587

Giffhorn H, Rampinelli A, Bonatelli Filho L, Collaço J - Acquired thrombocytopenia and cardiac surgery: case report. Rev Bras Cir Cardiovasc 2002; 17(2): 57-61.

ABSTRACT: Thrombocytopenia is a major problem in patients undergoing cardiac surgery. We will report two surgical cases with acquired decrease of platelets: one due uremia and other of a valvar dysfunction. These patients had an uneventful recovery in the transoperatory and immediately post-operatory phases. Blood loss was not higher than the expectected. Transfusion of platelets helded in the surgery could prevent future complications in the early recovery of the patient .

DESCRIPTORS: Thrombocytopenia, surgery. Blood platelets, pathology. Blood coagulation. Hemostasis.

\section{REFERÊNCIAS BIBLIOGRÁFICAS}

1. Campbell FW, Edmunds Jr H - In: Gravlee GP, Davis RF, Utley JR, eds. Cardiopulmonary bypass: principles and practice. Baltimore: Willians \& Wilkins, 1993:407-35.

2. Rapaport SI - Introdução à hematologia. São Paulo: Editora Harper \& Row do Brasil, 1978: 276-92.

3. Bithell TC - In: Lee GR, Bithell TC, Foerster J., Athens JW, Lukens JN, eds. Wintrobe's clinical hematology. Philadelphia: Lea \& Febiger, 1993: 135662.

4. Carvalho AC - Acquired platelet dysfunction in patients with uremia. Hematol Oncol Clin North Am 1990; 4:129-43.

5. Blauhut $B$, Harringer $W$, Bettelheim $P$, Doran JE, Späth $P$, Lundsgaard-Hansen $P$ - Comparison of the effects of aprotinin and tranexamic acid on blood loss and related variables after cardiopulmonary bypass. J Thorac Cardiovasc Surg 1994; 108: 108391.

6. Messmore HL, Nand S, Godwin J - In: Pifarré $\mathrm{R}$, ed. Anticoagulation, hemostasis, and blood preservation in cardiovascular surgery. Philadelphia: Hanley \& Belfus, 1993: 185-200.

7. Westphal K, Martens S, Strouhal U et al. Heparin-induced thrombocytopenia type II: perioperative management using danaparoid in a coronary artery bypass patient with renal failure. Thorac Cardiovasc Surg 1997; 45: 318-20.

8. Warkentin TE, Levine MN, Hirsh J et al. Heparin-induced thrombocytopenia in patients treated with low-molecular-weight heparin or unfractionated heparin. N Engl J Med 1995; 332: 1330-5.
9. Rosenthal DS \& Braunwald E. In: Braunwald E, ed. Heart disease: a textbook of cardiovascular medicine. Philadelphia : WB Saunders Company, 1992: 1742-66.

10.Christiansen S, Jahn UR, Meyer J et al. Anticoagulative management of patients requiring left ventricular assist device implantation and suffering from heparin-induced thrombocytopenia type II. Ann Thorac Surg 2000; 69: 774-7.

11. Sodian R, Loebe M, Gorman KF, Riess H, Hetzer R - Heparin induced thrombocytopenia: experiences in 12 heart surgery patients. ASAIO J 1997; 43: M430-3.

12. Doherty DC, Ortel TL, Bruijn N, Greenberg CS, Van Trigt 3rd - "Heparin-free "cardiopulmonary bypass: first reported use of heparinoid (ORG 10172) to provide anticoagulation for cardiopulmonary bypass. Anesthesiology 1990; 73: 562-5.

13.Peters FP - Treatment of heparin-induced thrombocytopenia. J Thorac Cardiovasc Surg 1997; 114: $517-8$.

14.Kappa JR, Ellison N, Fischer CA, Addonizio VP - The use of iloprost (ZK36374) to permit cardiopulmonary bypass in 2 patients with heparininduced thrombocytopenia. Anesthesiology 1985; 63(3 A):A 32.

15.Kappa JR, Fischer CA, Todd B et al. Intraoperative management of patients with heparininduced thrombocytopenia. Ann Thorac Surg 1990; 49: 714-23.

16.Zulys VJ, Teasdale SJ, Michel ER et al. - Ancrod (Arvin) as an alternative to heparin anticoagulation for cardiopulmonary bypass. Anesthesiology 1989; 71: 870-7. 
17. Teasdale SJ, Zulys VJ, Mycyk T, Baird RJ, Glynn MF - Ancrod anticoagulation for cardiopulmonary bypass in heparin-induced thrombocytopenia and thrombosis. Ann Thorac Surg 1989; 48: 712-3.

18. O-Yurvati AH, Laub GW, Southgate TJ, McGrath LB - Heparinless cardiopulmonary bypass with ancrod. Ann Thorac Surg 1994; 57: 1656-8.

19. Arnoletti JP \& Whitman GJ - Heparin-induced thrombocytopenia in coronary bypass surgery. Ann Thorac Surg 1999; 68:576-8.

20. Riess FC, Potzsch B, Bader R et al. - A case report on the use of recombinant hirudin as an anticoagulant for cardiopulmonary bypass in open heart surgery. Eur J Cardiothorac Surg 1996; 10: 386-8.

21. Koster A, Loebe M, Hansen R et al. - A quick assay for monitoring recombinant hirudin during cardiopulmonary bypass in patients with heparininduced thrombocytopenia type II: adaptation of the ecarin clotting time to the act II device. J Thorac Cardiovasc Surg 2000; 119:1278-83.
22. Koster A, Kuppe H. Hetzer R< mSodian R, Crystal GJ, Mertzlufft F - Emergent cardiopulmonary bypass in five patients with heparin-induced thrombocytopenia type II employing recombinant hirudin. Anesthesiology 1998; 89: 777-80.

23. Greinacher A, Völpel H, Janssens $U$ et al. Recombinant hirudin (lepirudin) provides safe and effective anticoagulation in patients with heparininduced thrombocytopenia: a prospective study. Circulation 1999; 99: 73-80.

24. McCrae KR \& Cines DB. In: Loscalzo J, and,Schafer Al, eds. Thrombosis and hemorrhage. Boston: Blackwell Scientific Publications, 1994: 54573.

25. Hursting MJ, Alford KL, Becker CP et al. Novastan $r$ (brand of argatroban): a small-molecule, direct thrombin inhibitor. Semin Thromb Hemost 1997; 23:503-16.

26. Addonizio VP, Fischer CA, Kappa JR, Ellison $\mathrm{N}$ - Prevention of heparin-induced thrombocytopenia during open heart surgery with iloprost (ZK36374). Surgery 1987; 102: 796-807. 